\title{
Medicinas tradicionales andinas y su despenalización: entrevista con Walter Álvarez Quispe
}

\author{
Decriminalizing traditional Andean medicine: \\ an interview with Walter Álvarez Quispe
}

Entrevista con

Walter Álvarez Quispe

Presidente vitalicio/Sociedad Boliviana de Medicina Tradicional. Avenida Franco Valle, 36 - zona 12 de octubre entre calles 1 y 2 El Alto - La Paz - Bolivia alvarezkallawaya@hotmail.com

\section{Concedida a}

\section{Carmen Beatriz Loza}

Directora de investigación, Instituto Boliviano de Medicina Tradicional Kallawaya.

Calle Rodrigo de Mejía, 1992

Miraflores - La Paz - Bolivia

cbeatriz.loza@gmail.com
LOZA, Carmen Beatriz. Medicinas tradicionales andinas y su despenalización: entrevista con Walter Álvarez Quispe. História, Ciências, Saúde - Manguinhos, Rio de Janeiro, v.21, n.4, out.-dez. 2014, p.1475-1486.

\section{Resumen}

Walter Álvarez Quispe, terapeuta kallawaya y biomédico especializado en cirugía general y ginecología, presenta la lucha de los terapeutas tradicionales y alternativos por la depenalización de estos sistemas médicos andinos realizada entre 1960 y 1990 . Bolivia se torna el primer país en América Latina y el Caribe en despenalizar la medicina tradicional antes de los planteamientos de la Conferencia Internacional sobre Atención Primaria de Salud (Alma-Ata, 1978). Los datos aportados por el entrevistado aseguran que los logros alcanzados, principalmente por los kallawayas, responden a un proyecto propio y autónomo. Estas conquistas no se deben a las políticas oficiales de interculturalidad en salud, aunque busquen atribuirse para sí los logros alcanzados.

Palabras clave: despenalización; medicinas tradicionales; kallawaya; medicinas alternativas; Bolivia.

\section{Abstract}

Walter Álvarez Quispe, a Kallawaya healer and biomedical practitioner specializing in general surgery and gynecology, presents the struggle of traditional and alternative healers to get their Andean medical systems depenalized between 1960 and 1990. Bolivia was the first country in Latin America and the Caribbean to decriminalize traditional medicine before the proposals of the International Conference on Primary Health Care (Alma-Ata, 1978). The data provided by the interviewee show that the successes achieved, mainly by the Kallawayas, stem from their own independent initiative. These victories are not the result of official policies of interculturality in healthcare, although the successes achieved tend to be ascribed to them.

Keywords: decriminalization; traditional medicine; Kallawaya; alternative medicine; Bolivia. 
$\mathrm{L}^{\mathrm{a}}$ as denominadas medicinas tradicionales siguen siendo claves en el desarrollo sanitario de América Latina y el Caribe. El impresionante incremento de la demanda de alternativas terapéuticas ajenas al sistema biomédico nos obliga a estudiar la historia de la despenalización de las medicinas tradicionales como claves para entender lo que acontece hoy en día en ese campo. Con ese propósito entrevistamos a Walter Álvarez Quispe, nacido en 1940, terapeuta indígena kallawaya, ${ }^{1}$ quien además tiene la particularidad de ser especialista en cirugía y ginecología graduado en la Universidad de la Habana, Cuba (1970), y fundador del Instituto de Ciencias Básicas y Preclínicas Victoria de Girón. Además, posee una larga trayectoria en instituciones cubanas como el Hospital Universitario Calixto García, y cuenta con el reconocimiento de los miembros del Hospital Clínico Quirúrgico Diez de Octubre. ${ }^{2}$ Esas dos formaciones - en medicina tradicional y occidental - y los numerosos reconocimientos internacionales recibidos durante su carrera han sido notables para su intervención directa en el desarrollo de la defensa de las medicinas tradicionales andinas desde la Confederación Única de Trabajadores Campesinos de Bolivia (1979) y el Parlamento de la República de Bolivia como Diputado por La Paz (1982-1993). ${ }^{3}$ El acopio de información acerca de su experiencia sirve para comprender mejor la dinámica interna del proceso, además, de conocer la intervención de individuos cuyo accionar da cuenta de los proyectos y las iniciativas autónomas bolivianas.

Esta entrevista, realizada en la ciudad boliviana de El Alto, nos ofrece la oportunidad de acercarnos al problema de la despenalización de las medicinas tradicionales desde un actor social que nos introduce a la cara no oficial de la historia para ahondar en el programa, la lucha y el trabajo realizado por los terapeutas tradicionales, tanto antes como después de la Conferencia Internacional sobre Atención Primaria de Salud de Alma-Ata (1978) que reivindicó algunas de las bondades de este recurso médico. ${ }^{4}$ Examinar esa narrativa testimonial significa descentrar la mirada histórica que enfatizó en el impacto de las propuestas de la Organización Mundial de la Salud (OMS). Esta mirada ha tenido como efecto pernicioso invisibilizar los esfuerzos locales de los terapeutas tradicionales realizados independientemente desde las periferias suramericanas mucho antes del planteamiento global de la OMS. Una mirada en parte confundida por el hecho que las directrices de esta institución sirvieron de

\footnotetext{
${ }^{1}$ El termino Kallawaya tiene varios significados, por un lado, alude a un grupo étnico ubicado en la actual Provincia Bautista Saavedra; por el otro, a los médicos itinerantes originarios de esa etnia cuyo saber médico, ritual y herbolario ha sido transmitido en el seno del grupo, de generación en generación, en el seno de los linajes kallawayas.

${ }^{2}$ Este dato es confirmado por el diploma otorgado al entrevistado en la Habana el 17 de octubre de 2012 por Fidel Castro Ruz.

${ }^{3}$ La información es corroborada por los documentos notariales que existen en la unidad del Ministerio de Justicia: la Comisión Técnica de Calificación de los Perseguidos de las Dictaduras, 2010. Con relación a los reconocimientos resaltamos el Certificado al Mérito de la Unión de Practicantes de Africa. Asímismo, la condecoración entregada en España como practicante de las medicinas naturales alternativas (1988), entre más de medio centenar de condecoraciones recibidas.

${ }^{4}$ La Declaración de Alma Ata fue elaborada durante la Conferencia Internacional sobre Atención Primaria de Salud que tuvo lugar en la Unión de Repúblicas Socialistas Soviéticas del 6 al 12 de septiembre de 1978. Esta conferencia internacional sobre atención primaria de salud promueve la incorporación de "médicos, enfermeras, parteras, auxiliares y trabajadores de comunidad, así como de personas que practican la medicina tradicional, en la medida que se necesiten, con el adiestramiento debido en lo social y en lo técnico, para trabajar como un equipo de salud y atender las necesidades de salud expresas en la comunidad" (OMS, 2002, p.23).
} 
apoyo para presentar las reivindicaciones de los terapeutas tradicionales ante sus estados. Al mismo tiempo, esta mirada no siempre fue útil para mostrar intentos de diálogo pasados entre sistemas médicos que se realizaron en condiciones desventajosas para las medicinas tradicionales por carecer de un total respaldo legal.

Este tema sigue siendo pertinente porque la subregión andina todavía es percibida como un espacio de muy baja regulación debido a que no existen registros sistemáticos de los terapeutas ni de sus instituciones y menos aún de sus prácticas debido a que no todos los sistemas médicos forman parte de los sistemas de salud oficiales (Nigenda et al., 2001). Este vacío en políticas y sistemas articulados de salud, ha dado pie recientemente a la implementación de mecanismos jurídicos para la institucionalización estatal de las medicinas tradicionales con la consiguiente aplicación de una política común en la subregión. De hecho, en las nuevas constituciones políticas del Estado, en Ecuador (2008) y Bolivia (2009), se incorporó la medicina tradicional a los sistemas de salud de ambos países. Es importante señalar que ello ocurrió al mismo tiempo que se reconoció la importancia de la interculturalidad en salud como política de Estado. Por ejemplo, en Bolivia se creó un Viceministerio de Medicina Tradicional e Interculturalidad que representa exclusivamente los intereses del Estado en ese campo (Bolivia, 8 mar. 2006).

La comprensión de la historia inmediata de la institucionalización estatal de las medicinas tradicionales se halla también relacionada con la lucha por la despenalización, pues los sistemas médicos andinos se mantuvieron ilegal o clandestinamente durante siglos. Lo paradójico es que los numerosos estudios sobre movimientos indígenas andinos no incluyeron en sus análisis las luchas emprendidas para mantener los saberes médicos tradicionales. La tendencia historiográfica dominante ha consistido en referirse exclusivamente a las preocupaciones de los indígenas y campesinos únicamente por los problemas de tierra y territorio o el control de los recursos renovables para el mercado. Por ello es urgente establecer una corrección que permita conocer las dinámicas de los terapeutas tradicionales por vencer a la legislación adversa, inaugurada por el colonialismo español en el siglo XVI y mantenida por cientos de años en América Latina y el Caribe. Al mismo tiempo, es necesario delimitar cronológicamente las coyunturas internas del proceso que permanecen todavía inciertas, así como su pertinencia para comprender la historia en los sistemas médicos en la subregión andina.

Un balance bibliográfico nos permite identificar matices en las lecturas e interpretación de los hechos históricos que envuelven la historia de las medicinas tradicionales y alternativas andinas; en primer lugar, aquellas que aceptan 1978 como el momento fundador para un denominado "reconocimiento" de la medicina tradicional. Sobre todo destacan las narraciones de biomédicos y químicos que se apoyan en las citas y en la reproducción de las resoluciones, guías y estrategias de la OMS (Sánchez Garrafa, Sánchez Garrafa, 2009; Loza, 2014; Fernández Ibarguen, 2009; Gómez Paye, 2001; Campos Navarro, 1997). Complementan esa perspectiva aquellos estudios que buscaron determinar el grado de integración, coexistencia y tolerancia de la medicina tradicional en América Latina y el Caribe (Nigenda et al., 2001). También sobresalen los ensayos que analizan las iniciativas desarrolladas por los terapeutas tradicionales entre 1960 y 1990, como si sólo fueran parte de los procesos de las actuales políticas de salud intercultural implementadas en la subregión (p. ej., Fernández Juárez, 2010; Baxeiras Divar, 2004). 
Históricamente, la despenalización corresponde a un proceso distinto al implementado por la interculturalidad en la salud iniciada hace pocos años. Hacemos notar que la intensión de entrelazar la medicina tradicional con las politicas estatales interculturales no es políticamente neutra. Los interculturalistas funcionales al sistema biomédico argumentan que la existencia de su proyecto de interculturalidad en la salud está engarzado con las luchas sociales previas de los terapeutas tradicionales. Esa continuidad histórica es ficticia como lo veremos a lo largo de la entrevista. Un ejemplo de esa narrativa del psiquiatra español José Luís Baxeiras Divar (2004, p.23) en Salud intercultural en Bolivia a inicios del tercer milenio donde sostiene que las "acciones en salud intercultural en Bolivia en los últimos tiempos" tendrían como origen el período comprendido entre 1960 y 1980. Al analizar con cuidado los documentos, planteamos que esta afirmación no tiene asidero. El concepto antropológico de interculturalidad en salud impregna el vocabulario de las autoridades de salud andinas tardíamente. Basta revisar el Código sanitario de la República de Bolivia (Bolivia, 1958) y la ley n.15.629 que aprueba ese código, para comprobar que no existe mención alguna de la interculturalidad. En realidad, sólo a partir del 2000 la cooperación internacional introduce el concepto de interculturalidad en salud, promoviéndolo, imponiéndolo e intentando aplicarlo durante los gobiernos bolivianos neoliberales sin resultados consistentes. Posteriormente, la interculturalidad se estatizó en Ecuador y Bolivia y cobró plena vigencia con la formulación de las nuevas constituciones, que generaron un cuerpo de disposiciones donde se incorporó la noción profusamente a pesar, claro está, de la opacidad del propio concepto que no termina de posarse en el mundo andino o que simplemente asume múltiples significados, algunos de ellos mermando la potencialidad crítica del proyecto intercultural.

En la bibliografía sobre salud intercultural no se reconoce plena y explícitamente que existió una alianza entre indígenas y no indígenas para realizar acciones colectivas de resistencia, movilización y lucha legal para intentar desterrar la penalidad colonial sobre sus saberes y prácticas médicas. Menos se toma en cuenta por los estudiosos que, en Bolivia, entre 1977 y 1990, se dieron y realizaron, por primera vez, en América Latina, los primeros pasos para alcanzar la legalidad de las medicinas tradicionales lo que conduciría a la parcial despenalización. En la Tabla 1 presentamos la frecuencia de actividades desarrolladas

Tabla 1: Frecuencia de las actividades desarrolladas por los terapeutas tradicionales para la despenalización de la medicina natural, cultura aymara y quechua desde 1973 hasta 1979

\begin{tabular}{|l|c|r|c|r|c|c|}
\hline Frecuencia & Actividades & $\%$ & Días trabajados & $\%$ & Participantes & $\%$ \\
\hline Congresos* & 8 & 10,7 & 46 & 15,6 & 636 & 12,6 \\
\hline Seminarios & 1 & 1,3 & 5 & 1,7 & 82 & 1,6 \\
\hline Cursos & 7 & 9,3 & 62 & 21 & 620 & 12,2 \\
\hline Cursillos & 35 & 46,7 & 127 & 43,1 & 1018 & 20,5 \\
\hline Conferencias & 12 & 16 & 13 & 4,4 & 2707 & 53,5 \\
\hline Difusión** & 12 & 16 & 42 & 14,2 & - & - \\
\hline Total & 75 & 100 & 295 & 100 & 5063 & 100 \\
\hline
\end{tabular}

Fuente: elaborado por la autora sobre la base del informe del catequista y promotor Rufino P"axsi Limachi a la Novena Asamblea de Medicina Natural, La Paz, 26 de noviembre de 1979 (Medicina..., 1980).

* En 1976 se desarrolló el congreso en Puno, República del Perú, contando con 240 asistentes, superando el efectivo de personas congregadas en Bolivia.

** Se trata de 12 programas realizados en Radio Progreso (La Paz) que se difundieron durante 42 días con duración de 42 horas. 
por indígenas y campesinos del altiplano y valles para lograr visibilidad y posicionando públicamente la temática en Bolivia y Perú a través de numerosas actividades desarrolladas desde 1973 hasta 1979.

Ofrecer datos precisos es de suma importancia, porque actualmente se difunde la idea errónea que fueron las instancias biomédicas del Estado las propiciadoras de ese movimiento, otras veces no reconocen la verdadera autoría de los médicos tradicionales o simplemente se minimiza su alcance como epifenómenos de decisiones de instituciones oficiales (p. ej. Bolivia, 2011, p.54). Al mismo tiempo, algunos autores pasan por alto y llegan a ocultar datos acerca de la resistencia desarrollada por las universidades, los colegios médicos y colegios de farmacia y bioquímica bolivianos que dificultaron y retardaron la aprobación de muchas leyes que favorecían la libre práctica de las terapias tradicionales y alternativas (p. ej., Bolivia, 2011; Baxeiras Divar, 2004, p.23).

La despenalización de las medicinas tradicionales fue un proceso autónomo boliviano que tuvo repercusiones a nivel latinoamericano, particularmente en la formas de organización legal de los terapeutas tradicionales. La entrevista nos ilustrará sobre el desarrollo de este proceso.

Carmen Beatriz Loza

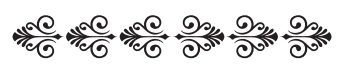

¿Por qué hablar de despenalización de las medicinas tradicionales andinas y no de su reconocimiento?

Las medicinas tradicionales andinas hasta hace muy poco tiempo existían penalizadas. En Bolivia estuvieron oficialmente prohibidas desde el siglo XVI hasta la mitad del siglo XX y hasta mucho más tarde en el resto de países de América Latina y el Caribe. Las instituciones oficiales de salud biomédica no reconocían el trabajo de los terapeutas tradicionales porque simplemente negaban su existencia, amparados en disposiciones jurídicas. Sencillamente, cerraban los ojos ante su empleo por amplios sectores de la población y, obviamente, les negaban los aportes realizados a la salud pública de Sudamérica. Mientras tanto, la población utilizaba la medicina tradicional en la clandestinidad y con todos los riesgos que eso implicaba para los pacientes. En parte se debía a que el sistema de salud boliviano adolecía de debilidades, pues no abarcaba todo el territorio por el mismo hecho que la población se hallaba muy dispersa y poco articulada por los sistemas de comunicación internos. Además, contaba con una institucionalidad en salud precaria, porque el país tuvo un Ministerio de Salud sólo en 1936, después de la Guerra del Chaco, que enfrentó a Bolivia con el Paraguay entre 1932 y 1936.

Siguiendo el hilo conductor de la Guerra del Chaco, ¿por qué los terapeutas kallawayas que estuvieron en el frente de batalla, atendiendo las enfermedades y heridas de la tropa indígena, no fueron reconocidos por su aporte a la salud pública?

Al sistema biomédico le costaba entender que los kallawayas realizaron aportes a la salud pública de Sudamérica. Su actitud osciló entre la negación de nuestra medicina y la utilización 
oportunista, coyuntural e inmediatista de los servicios de los terapeutas tradicionales. Durante la Guerra del Chaco se requirió del servicio de los kallawayas porque no había suficientes biomédicos y tampoco había medicamentos. Allí, en pleno campo de batalla, se objetivaron los conocimientos médicos y herbolarios kallawayas, porque fueron ellos los que realizaron esfuerzos por salvar a los indígenas que morían, sobre todo de sed, en un calor abrasador. Con la ayuda de plantas y animales que conocían, hacían cataplasmas, emplastos y brebajes. En ese afán perdimos un importante contingente de kallawayas, tal como me lo narró mi padre Lorenzo Álvarez (1907-2005) y mis tíos Antonio Álvarez Mamani (1912-1997) y Justiniano Mamani (1912-1992).

Esta guerra les sirvió para tomar contacto con personajes que posteriormente tuvieron una participación importante en la política boliviana, por ejemplo, los que vendrían a ser presidentes de Bolivia: Hernán Siles Suazo (1913-1996) y Víctor Paz Estenssoro (1907-2001). Este último personaje tuvo un peso gravitante en el destino de la medicina tradicional, pues en su administración gubernamental se logró aprobar disposiciones jurídicas importantes que fueron pioneras a nivel sudamericano, entre ellas, la aceptación del empleo de la medicina tradicional y de su investigación. Estas decisiones fueron tomadas porque él fue testigo ocular del trabajo específico de los kallawayas en el servicio a los enfermos en tiempos de guerra. Con ello quiero dejar establecido que la relación de los kallawayas con Víctor Paz Estenssoro no se debe a vínculos estrictamente político-partidarios como erróneamente se ha tratado de sustentar. Los lazos con Víctor Paz Estenssoro se entablaron en tiempos de guerra, es decir cuando era soldado raso en la Batería Seleme y más tarde, interventor de la Contraloría General en el Cuerpo del Ejército.

La objetivación del conocimiento médico kallawaya, es decir la prueba de curaciones exitosas hechas públicas, se había producido mucho antes de la Guerra del Chaco. ¿Consideras que existía una memoria larga de esos momentos públicos de curaciones exitosas?

Sin lugar a dudas que sí. En mi comunidad, Canlaya, desde niño escuché narraciones de las curaciones (en sentido amplio) que realizaron mis familiares, por ejemplo, cuando se estaba construyendo el Canal de Panamá. Supe que alrededor de 1888 se presentó un fuerte brote de malaria que estaba causando la muerte de los trabajadores latinoamericanos y del Caribe que fueron a brindar su fuerza de trabajo a las compañías francesas y estadounidenses. Entre ellos, algunos peruanos que estaban en las obras propusieron encontrar una solución contratando kallawayas. Los peruanos descendieron desde Panamá hasta el pueblo Charasani, precisamente a mi comunidad, Canlaya (el único lugar que visitaron). Allí fueron alojados en casa de mi abuelo, Dionisio Álvarez, donde se realizaron reuniones para explicar los síntomas y signos que padecían los trabajadores y determinar la patología a partir de los rasgos que fueron expuestos. Un grupo de kallawayas planteó la hipótesis que se trataba de chujchu, el nombre quechua de la malaria. Inmediatamente organizaron el viaje hasta Panamá, cargados de dos plantas importantes del norte de La Paz: coca (Erythroxylum coca) y quina (Cinchona calisaya y otras especies de Cinchona). Cargados de esas plantas, un grupo de kallawayas junto a los peruanos realizaron la travesía desde mi comunidad vía Lima y desde allí hasta detenerse en Panamá. Sabemos que en el Canal en construcción prepararon infusiones de coca y quina para que beba tanto la población afectada como aquella que no tenía síntomas. 
La curación fue exitosa y muy comentada en su época. De ese viaje se conserva una fotografía que fue recuperada por el antropólogo francés Louis Girault (1921-1975). Como tú bien lo has señalado, ${ }^{5}$ la curación de Panamá fue clave para que el gobierno de Aniceto Arce (1824-1906) obligue a los kallawayas a preparar un herbario que fue mostrado en la Exposición Universal de Paris, entre 1889 y 1891, mereciendo una mención en la premiación final.

¿Qué pasaba en los años 1970 en el campo de la medicina tradicional; algo había cambiado desde que te fuiste a Cuba en 1962?

Cuando regresé de Cuba, después de hacer mis estudios biomédicos, me reencontré con mis parientes kallawayas y recordé acerca mi infancia con ellos, sobre todo, junto a mis tíos: Antonio Álvarez Mamani, Ramón Álvarez, Justiniano Mamani, quienes me encaminaron desde la niñez a la atención de pacientes en las minas bolivianas de Oruro y Potosí. Ellos me comunicaron que a fines de los años 1970 seguían curando en clandestinidad y sufriendo los abusos de las autoridades y la policía, quienes los perseguían, hostigaban y privaban de libertad de su práctica. A pesar de ello, algunos kallawayas estaban en la ciudad de La Paz cerca de la Iglesia de San Francisco. Allí se ubicaron en vista de que en esos años estaban impedidos de ingresar a Tiwanaku, uno de los centros ceremoniales más importantes del altiplano por su condición de sitio arqueológico y que está ubicado a 29 kilómetros de la ciudad. Más allá de ello, me sorprendió que, pese a esos "cercos", los kallawayas siguieran viajando al Perú, Argentina y Chile, pues en esos países tenían pacientes y curaban en forma clandestina.

Los kallawayas mayores me encargaron que

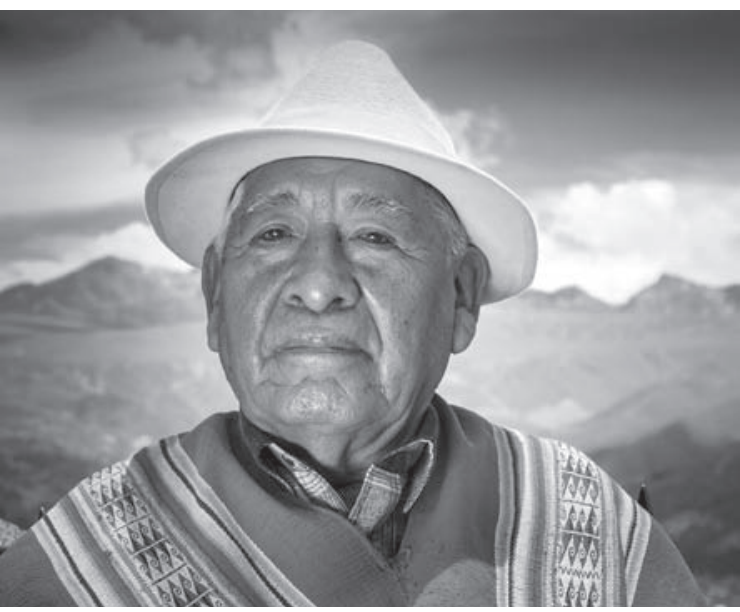

Figura 1: Kallawaya y biomédico Walter Álvarez Quispe en El Alto, 2014 (Fotografía Francisco Xavier Loza) cambiara esa situación insostenible y haga reconocer la medicina que había practicado este pueblo durante siglos. Inicialmente quisieron nombrarme ejecutivo de la Provincia Bautista Saavedra, un cargo de máxima dirección de las comunidades kallawayas. Luego me mandaron como su representante a la Federación de Campesinos Tupaj Katari en una coyuntura muy difícil, pues esta institución era dominada y controlada por los campesinos aliados al dictador Hugo Banzer Suárez (1926-2002). Por esa razón, trabajé en la fundación de la Confederación Sindical Única de Trabajadores Campesinos de Bolivia, junto a muchos célebres dirigentes con quienes revertimos esa dominación política. Una vez creada la institución, ocupé el cargo de primer secretario de Salud y me propuse despenalizar las medicinas tradicionales con la ayuda de ese movimiento. Eso me pareció más viable que la defensa de la medicina natural iniciada en 1965. A propósito, deseo aclarar que en esa época y todavía hoy, el término de medicina natural se utiliza en los medios urbanos como bolivianismo para señalar a los terapeutas que manejan recursos herbarios y no tiene el sentido de naturismo formado en

${ }^{5}$ Ver Loza (2004). 
escuelas especializadas porque son inexistentes en el país, a diferencia del resto de países de América Latina y el Caribe.

A tu llegada a La Paz en 1977 te incorporas a un movimiento de defensa de las medicinas naturales, organizado en 1965. Específicamente, participas en la novena Asamblea Boliviana en Medicina realizada en La Paz en la iglesia de María Auxiliadora en noviembre de 1979 ¿Qué rol te toca jugar durante ese evento?

En 1965, en la zona de Tiwanaku, la Conferencia Episcopal Boliviana encomendó al jesuita boliviano, Jaime Zalles Asín (?-2007), la organización de la Asamblea Boliviana en Medicina, la cual fue exitosa, llegando a organizarse ocho eventos de ese tipo: siete en el altiplano de La Paz (1973-1975 y 1977-1978) y uno en el Perú (1976). En la novena asamblea (1978), participan un total de treinta personas. Recuerdo que predominaban aymaras (60\%), pero también asistían no indígenas (27\%) y kallawayas (13,3\%). El propósito consistía en avanzar en la defensa de la medicina natural con un sustento bíblico. Aclaro ese aspecto, pues el objetivo era transmitir conocimientos a través de 75 actividades (ver Tabla 1) para la instalación de escuelas para la formación de "sanitarios de medicina natural". No se trató de mantener y fortalecer a los terapeutas tradicionales con sus formas propias de entender la salud, atención y enfermedad, debido a su antiguo contenido religioso andino. Me llamó mucho la atención la falta de trabajo en el reconocimiento jurídico después de ocho asambleas realizadas desde 1973 y la falta de avances al respecto, a pesar de que eso era clave en ese momento. Había que frenar la persecución sustentada en las disposiciones que estaban vigentes en las leyes bolivianas.

Los planteamientos no fueron claros en esa época, pues existía temor de reclamar a la ministra de Salud, Aida Claros de Bayá, presente en el evento, acerca de la situación de ilegalidad en la que se encontraban las medicinas tradicionales y las alternativas en Bolivia. En esa ocasión apenas se le entregó una carta donde se planteó dos aspectos: el primero, señalándole la existencia de una evidente pugna médica responsable de un "diálogo imposible" entre

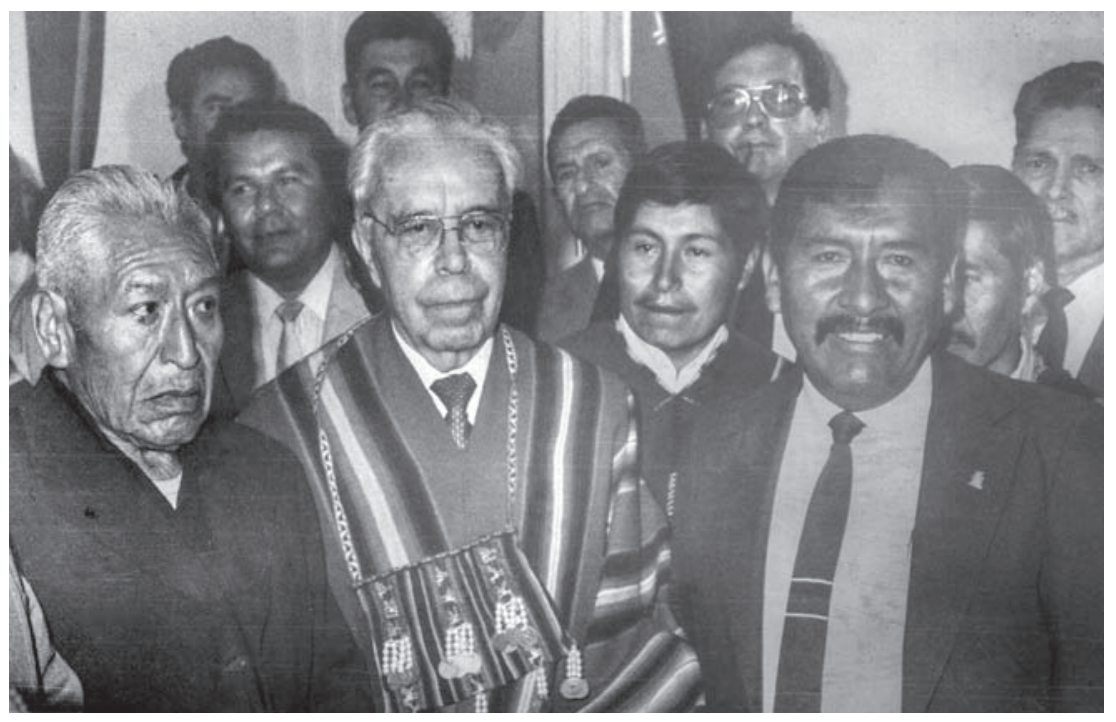

Figura 2: A la izquierda kallawaya René Llaves; al centro, con poncho, el doctor Victor Paz Estensoro, presidente de la República de Bolivia; a la derecha kallawaya. René Llaves hijo y kallawaya Walter Álvarez Quispe. En el Palacio de Gobierno de La Paz, 1987 (Archivo privado de Walter Álvarez Quispe) 
sistemas médicos al recordarle la existencia de una medicina kallawaya en Bolivia, la cual era negada desde tiempos coloniales, y el segundo, planteándole la integración de las líneas directrices de la OMS con el fin de obtener facilidades para la apertura y funcionamiento de consultorios públicos de medicinas tradicionales y alternativas. Conseguir la autorización era una forma de probar que existía apertura, como muestran los documentos cuyos principios se basaban en "Mantener una relación de mutuo respeto entre los agentes de medicina oficial y los de medicina tradicional popular". ${ }^{6}$

En ese sentido, mi intervención consistió en legalizar la medicina tradicional y fortalecer la creación de una institución que concentre legalmente a todos los terapeutas en una época en la cual había persecución (incluidos asesinatos y desapariciones forzadas de terapeutas porque eran considerados brujos). Esa institución es la Sociedad Boliviana de Medicina Tradicional, la primera y más antigua en América Latina y el Caribe. Dirigí esa institución durante dos décadas por elección democrática y por presión de las bases, pero sobre todo en momentos difíciles y de amenaza permanente a la integridad institucional. Dispuse de mi propio capital y fuerza intelectual para mantener la institución cuando era difícil sostenerla en términos materiales. Participé política y partidariamente para lograr posicionar la problemática de las medicinas tradicionales en el parlamento, siendo éste un hecho inédito en su época. Todavía muchos parlamentarios se negaban a tratar la temática, pero logré posicionarla por las curaciones que realicé a los propios diputados y senadores. Estos parlamentarios, una vez sanados de sus males, llegado el momento, tuvieron que sancionar las leyes que propuse en favor de la medicina tradicional. El cuerpo legal, en mi gestión parlamentaria, sirvió de sustento para la preparación del expediente, para que la Organización de las Naciones Unidas para la Educación, la Ciencia y la Cultura (Unesco) declare la cosmovisión kallawaya como obra maestra del patrimonio oral e intangible de la humanidad. De esa manera protegí nuestros conocimientos, sobre todo las terapias, ritualidad y manejo de los recursos herbáceos, que es una declaratoria patrimonial preparada bajo la dirección del Instituto Boliviano de Medicina Tradicional Kallawaya y del Viceministerio de Cultura.

En 1978, el Estado sugiere la incorporación de la medicina tradicional al sistema de salud nacional al influjo de la OMS. En esa época, los terapeutas tradicionales plantearon la "incorporación" o la "adaptación" de la biomedicina a nuestros sistemas médicos tradicionales porque la mayoría de la población seguía utilizando los recursos terapéuticos andinos o las medicinas alternativas. Es decir, estábamos reinvirtiendo el sentido de la propuesta de la OMS. Ese proceso local boliviano repercutió a nivel suramericano, por ejemplo, a partir de 1984, tomó contacto conmigo el famoso biomédico y neurocirujano Fernando Cabieses Molina (1920-2009) para recoger la experiencia e iniciar el proceso de despenalización en el Perú. Allí, los intelectuales tuvieron un papel protagónico, a diferencia de lo ocurrido en Bolivia, donde fueron los terapeutas indígenas que tomaron la dirección del proceso.

Se afirmó en un documento institucional que "Bolivia tiene el mérito de haber sido el primer país del continente que aceptó oficialmente la vigencia de las medicinas tradicionales. Posteriormente, en 1987, se fundó la Sociedad Boliviana de Medicina Tradicional. Sin embargo, fue recién en los

${ }^{6}$ Ver Medicina... (1980, p.8). 
años 1990 que se empezaron a escuchar propuestas de políticas y estrategias de articulación". ¿Qué opinión te merece esta afirmación?

Uno de los problemas más claros de la bibliografía es que los autores - en su mayoría extranjeros - no han participado de los procesos de despenalización y tampoco han colaborado en el mismo, de tal suerte que desordenan los eventos, las fechas y las condiciones históricas del proceso. A ello se suma el desconocimiento de las lenguas indígenas, lo cual los lleva a mayor confusión. De manera errónea atribuyen a las Organizaciones No Gubernamentales, a la Cooperación Internacional o al Estado todos los méritos de la despenalización. Presentan a los terapeutas tradicionales como pasivos, como si hubiésemos estado con los brazos cruzados, admitiendo la fatalidad de ver impedida nuestra práctica médica tradicional. En primer lugar, debo aclarar que la despenalización es un hecho colectivo y abanderado por los kallawayas, junto a terapeutas de diversas etnias y tradiciones médicas del país. A mí me tocó afrontar la lucha en dos planos: por un lado, trabajando para obtener una personería jurídica de la Sociedad Boliviana de Medicina Tradicional cuya creación fue en 1965, cobrando ésta la legalidad el 10 de enero de 1984 (con ello aclaro la fecha exacta), y por otro lado, encarando la lucha política y parlamentaria para exigir y plantear la existencia de un instituto de formación e investigación de las medicinas tradicionales. Se batalló para recuperar la medicina de los pueblos indígenas, no en vano hice declarar a la Provincia Bautista Saavedra como Capital de la Medicina Tradicional de Bolivia (1987). Estoy convencido que mi formación de biomédico fue un elemento clave para iniciar la discusión en el Colegio Médico y las Facultades de Medicina, Farmacia y Bioquímica de La Paz donde se afianzó la resistencia a las medicinas tradicionales. Sin embargo, aclaro que mi intervención forma parte de un mandato que me dieron los mayores de mi pueblo kallawaya, quienes me educaron para esa tarea, al igual que los campesinos e indígenas del país a quienes representé como su secretario de Salud de la Confederación Única de Trabajadores Campesinos de Bolivia desde 1979 hasta 1983.

Contrariamente a la afirmación citada en la pregunta, en 1978 se edificó el Centro de Medicina Natural Wanqullo en Tiwanaku. En 1982 éste empezó a funcionar, atendiendo a los pacientes de las comunidades. Ésta fue la primera experiencia para demostrar que la medicina tradicional seguía viva, tenía valor y era importante brindarla públicamente. Allí atendía como biomédico y como kallawaya. Posteriormente, entre 1983 y 1984, se estableció en la ciudad de La Paz un Consultorio Integrado donde trabajaban biomédicos, terapeutas tradicionales (kallawayas, amawt'as) y naturistas. Adicionalmente, en 1989, hubo la experiencia de un consultorio en Villa Copacabana donde trabajaba el biomédico y el terapeuta tradicional en conjunto, pero fracasó por el éxito alcanzado por este último. Todas esas experiencias anteriores a la interculturalidad de la salud son muestra de iniciativas de diálogo entre sistemas médicos, emprendidas por los bolivianos de manera autónoma.

Según el biomédico y antropólogo mexicano Roberto Campos Navarro, ${ }^{8}$ la Sociedad Boliviana de Medicina Tradicional "por desgracia agrupó no sólo a médicos indígenas sino también a grupos

\footnotetext{
${ }^{7}$ Ver Bolivia (2005, p.7).

${ }^{8}$ Ver Campos Navarro (1997, p.73).
} 
mestizos que practican naturismo, la acupuntura y otras formas curativas". ¿Es tan infortunada esta experiencia?

Es innegable que el proceso inicial de la despenalización congregó a una pluralidad étnica, que es el reflejo de la composición poblacional boliviana; además, reunió a practicantes de diversos sistemas y tradiciones médicas también presentes en la sociedad. No por esto existía razón para excluir y marginar a los mestizos nada más por no ser indígenas, pues son parte constitutiva de la sociedad boliviana. Por ejemplo, algunos mestizos que practicaban las curaciones herbáceas y difundían el vegetarianismo fueron perseguidos. Recuerdo sobre todo a Cupertino Maldonado, quien cumplió prisión por ello. La lucha no era sólo de los sistemas médicos indígenas, sino también de los sistemas alternativos y debía construirse un bloque con los practicantes de otras medicinas y terapias. Estaba en juego la defensa de la sobrevivencia de todas las prácticas no biomédicas y había que cerrar filas en tiempos de extrema confrontación en los campos médicos.

La Sociedad Boliviana de Medicina Tradicional se afianzó y se constituyó no solo en la primera institución en Bolivia sino en Suramérica. A principios del 2000 se empezó a fragmentar la institución para dar cuerpo a más de doscientas instituciones en todo el país, lo cual fue favorecido por la polarización política en el país y los intereses particulares de los miembros de la institución que encontraron una manera oportuna de seguir sus propios derroteros y sus propios proyectos acordes a la nueva coyuntura. El panorama había cambiado, después de una larga lucha, pero los objetivos históricos de la institución se cumplieron a cabalidad y la institución irradió más allá de nuestras fronteras. Con todo, conseguimos, por lo menos parcialmente, la conciencia de que el agrupamiento y la organización de los terapeutas tradicionales es un medio eficaz de lucha.

\section{REFERENCIAS}

BAXEIRAS DIVAR, José Luís.

Salud intercultural en Bolivia a inicios del tercer milenio. La Paz: Embajada de España en Bolivia; Cooperación Española; Embajada de España en Bolivia. 2004.

BOLIVIA.

Ministerio de Salud y Deportes. Mapeo de normas sobre salud materna y factores culturales en Bolivia. 1994-2010. La Paz: Ministerio de Salud y Deportes. 2011.

BOLIVIA.

Constitución Política del Estado. La Paz: Presidencia de la República. 2009.

BOLIVIA.

Decreto supremo n.28.361. Reglamento a la Ley de Organización del Poder Ejecutivo. Disponible en: http://www.lexivox.org/norms/BO-DS-28631. html. Acceso en: 3 nov. 2014. 8 mar. 2006.

BOLIVIA.

Ministerio de Salud y Deportes. Guías de Interculturalidad. La Paz: Ministerio de Salud y Deportes. 2005.
BOLIVIA.

Código sanitario de la República de Bolivia. La Paz: s.n. 1958.

CAMPOS NAVARRO, Roberto.

Curanderismo, medicina indígena y proceso de legalización. Revista Nueva Antropología, v.16, n.53-52, p.67-87. 1997.

ECUADOR.

Constitución política del Estado. Quito: Asamblea Constituyente. 2008.

FERNÁNDEZ IBARGUEN, Manuel.

La OMS y los sistemas médicos tradicionales. In: Sánchez Garrafa, Ricardo; Sánchez Garrafa, Rodolfo (Ed.). Medicina tradicional andina: planteamientos y aproximaciones. Cusco: Centro de Estudios Regionales Andinos Bartolomé de Las Casas. p.325-337. 2009.

FERNÁNDEZ JUÁREZ, Gerardo (Ed.).

Salud, interculturalidad y derechos: claves para la reconstrucción del Sumak Kawsay-Buen Vivir. Quito: Ministerio de Salud Pública. 2010. 
GOMEZ PAYE, Jesús.

Reglamentación de la práctica de la medicina tradicional. Tesis (Licenciatura en Ciencias Jurídicas y Políticas) - Facultad de Derecho Universidad de San Simón, Cochabamba. 2001.

LOZA, Carmen Beatriz.

¿Por qué es difícil subordinar la medicina tradicional andina a la biomedicina? História, Ciências, Saúde-Manguinhos, Rio de Janeiro, v.21, n.1, p.353-356. 2014.

LOZA, Carmen Beatriz.

Kallawaya: reconocimiento mundial a una ciencia de los Andes. La Paz: Unesco; Viceministerio de Cultura; Fundación del Banco Central de Bolivia. 2004.

MEDICINA...

Medicina natural. Pastoral social, v.3, n.6, p.1-13. 1980.
NIGENDA, Gustavo et al.

La práctica de la medicina tradicional en América Latina y el Caribe: el dilema entre regulación y tolerancia. Salud Pública de México, v.43, n.1, p.41-51. 2001.

SÁNCHEZ GARRAFA, Ricardo; SÁNCHEZ

GARRAFA, Rodolfo (Ed.).

Medicina tradicional andina: planteamientos y aproximaciones. Cusco: Centro Bartolomé de Las Casas; Centro de Medicina Andina. 2009.

OMS.

Organización Mundial de la Salud. Fondo de Socorro Internacional de las Naciones Unidas a la Infancia. Declaración de Alma-Ata. Salud y educación, v.1, n.1, p.22-24. 2002.

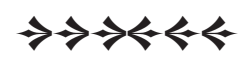

